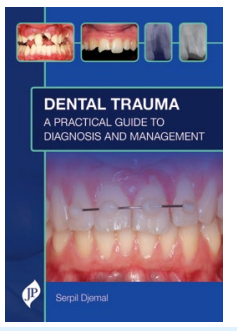

\section{DENTAL TRAUMA: A PRACTICAL GUIDE TO DIAGNOSIS AND MANAGEMENT}

Serpil Djemal;

2021; £72.00; JP Medical Publishers; pp. 146;

ISBN: 978-1909836662

The dilemma for a busy caring practitioner is that while the range of types of damage to the dental alveolar tissues is so wide, seeing any one type in practice can be so infrequent, then having to pause when confronted with an emergency involving trauma and having to think it out. Therefore, I found this book a very welcome and handy addition to update my knowledge to enable early and effective treatment of patients with damage to their teeth and associated structures from trauma.

A compact and practical guide, it is very comprehensive and gives clear instructions on how to assess, diagnose and effectively manage the most commonly encountered traumatic dental injuries a general practitioner is likely to see.

The well-defined chapters go through each type of possible trauma in a systematic manner with easy-to-follow protocols, which include step-by-step information. I like the way these protocols do not offer too much discussion, as the primary use is as a practical guide after all. However, for anyone seeking further background and/or discussion, there are adequate references at the end of each chapter.

The book covers trauma to the usual enamel, dentine, pulp, crown and root fracture injuries but has very helpful chapters on subluxation, extrusion, lateral luxation, intrusion and avulsion, continuing with dentoalveolar fractures and concluding with longterm consequences.

It is a small book but it is packed with over 400 high-quality photographs and relevant explanatory illustrations to support the case histories. It also discusses post-operative care and long-term management including splinting and root resorption.

The author, Serpil Djemal, has a huge amount of knowledge, experience and interest in the subject of dental trauma. She has been passionate enough to set up a charity - Dental Trauma UK. She has also managed to persuade a vast array of specialists, some better known than others, to make some very valuable contributions.

I am sure this book will make me much more confident in treating any dental trauma case that walks into the surgery from now on and help all other practitioners, at all stages of their careers, and all others involved in treating trauma, even in secondary care.

Russ Ladwa

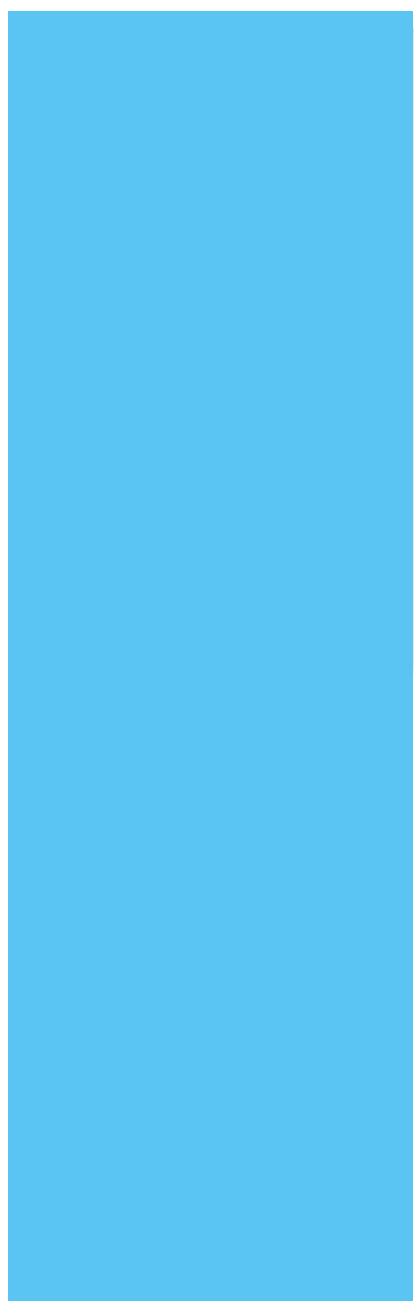

